\title{
A Note on Koch \& Schaechter's Hypothesis about Growth and Fission of Bacteria
}

\author{
BY E. O. POWELL \\ Microbiological Research Establishment, Porton Down, Salisbury, \\ Wiltshire
}

(Received 6 June 1964)

SUMMARY

The hypothesis of Koch \& Schaechter (1962) in its general form gives rise to mathematical relationships of great complexity. The formulae are simplified when certain conditions are met; the most important condition is that the smallest organism about to divide should not be smaller than the largest newly formed organism. Real cultures often satisfy the condition, and they can then fairly be used to test the hypothesis.

\section{INTRODUCTION}

Previous attempts to account for the variability of the generation times of individual bacteria have been based on the supposition that the generation time is directly determined by a nexus of primitive events, essentially chemical in nature and involving few molecules (Rahn, 1932; Kendall, 1948). In two classical papers (Adolph \& Bayne-Jones, 1932; Bayne-Jones \& Adolph, 1932) Adolph and BayneJones suggested that generation time was primarily determined by size at the time of fission; this suggestion seems to have been prompted only by the fact that, in the early stages of a batch culture, the mass growth rate begins to increase before the number growth rate, and, in the late stages, the mass growth rate decreases before the number growth rate. Koch \& Schaechter (1962) put forward a clearly formulated hypothesis of the same kind; they suppose that the primitive events, whatever their nature, determine primarily the size of the organism at its termination; the size (i.e. mass or volume) is a stochastic variable whose target is a certain critical value characteristic of a given species in a given environment. With the addition of a few natural and moderate assumptions, the pattern of generation times follows.

In their exposition Koch \& Schaechter make a number of approximations which they do not justify explicitly and quantitatively. The purpose of this present note is to give a more detailed account of the consequences of the hypothesis. It will appear that the hypothesis implies a pattern of growth involving mathematical relationships of great complexity, but that radical simplification is possible under certain conditions which are often met, with a measurable degree of error, by real cultures.

Koch \& Schaechter postulate, first, that the mass growth rate of the individual organism is a constant, say $\nu$, the same for all organisms in the steady state (I use $\nu$ instead of Koch \& Schaechter's $\lambda$ since the constant must, in the steady state, be equal to both the mass and number growth rate of the whole culture). The same will 
be true of the volume growth rate if the density remains constant, and less exactly of the length of rod-like organisms if both the density and diameter remain constant. In practice it is impossible to measure the mass or diameter with any accuracy, and I shall use $x$ to denote 'size' in the sense of mass, volume or length, leaving the appropriate interpretation for separate discussion or investigation. Then for any individual

$$
\frac{d x}{d t}=\nu x ; \quad x \propto e^{\nu t}
$$

Secondly, they postulate that the sizes of organisms at termination $\left(x_{t}\right)$ are scattered about a critical value, and that the $x_{t}$ are independent of one another. They assume further that the distribution of $x_{t}$ is normal, but this is an unnecessary and indeed unrealistic restriction. Thirdly, they postulate that when an organism divides the two daughter organisms are nearly equal in size. In some contexts, it is a reasonable approximation to assume that the daughters are exactly equal; in others the differences must be taken into account.

In the sequel I have varied Koch \& Schaechter's notation in order to avoid confusion with my own usage.

I make use of two principles which are not widely known:

(a) A convenient way is needed of determining the expectation $\left(\mu_{r}^{\prime}(\log v)\right)$ of the $r$ th power of the logarithm of a variate $v$ which takes only positive values. If $v$ has a frequency function $f(v)$ the Mellin transform

$$
\mathfrak{M}(s ; f)=\int_{0}^{\infty} v^{s-1} f(v) d v
$$

is a generating function for $\mu_{r}^{\prime}(\log v)$ in that

$$
\mu_{r}^{\prime}(\log v)=\left.\frac{d^{r}}{d s^{r}}\{\mathfrak{M}(s ; f)\}\right|_{s=1} .
$$

Similarly, $\log \{\mathfrak{M}(s ; f)\}$ is a generating function for the cumulants $\kappa_{r}(\log v)$. These statements are a simple transformation of, and are guaranteed by, equivalent statements which could be made in terms of the more conventional characteristic functions (Fourier complex transforms). The form given is direct in application since tables of Mellin transforms exist (Erdélyi, 1954). The Mellin transform differs from the ordinary moment-generating function of log $v$ only in having the index $s-1$ instead of $s$.

(b) If a variate $v$ takes only positive values, and its coefficient of variation is $c(v)$, then

$$
\operatorname{var} \log v \bumpeq c^{2}(v)
$$

provided (i) that $c(v)$ is small, (ii) that the higher moment ratios $\mu_{r} /\left(\mu_{1}^{\prime}\right)^{r}$ of $v$ are all small compared with $c(v)$.

\section{Inferences from Koch \& Schaechter's hypothesis}

\section{The preliminary difficulty}

Before proceeding, we must resolve a difficulty not discussed by Koch \& Schaechter. It appears as soon as we attempt to derive the generation-time distribution in a naïve way. 
Let $l\left(x_{t}\right)$ be the frequency function of the size of organisms at termination and put

$$
L_{-}\left(x_{t}\right)=\int_{x_{t}}^{\infty} l(\xi) d \xi
$$

Assume at first that sisters are exactly equal in size at inception $\left(x_{i}\right)$. Then the frequency function $l_{i}\left(x_{i}\right)$ of $x_{i}$ is $2 l\left(2 x_{i}\right)$. Write $f_{s}(\tau)$ for the frequency function of generation times $\tau$ and $F_{s-}(\tau)$ for

$$
\int_{\tau}^{\infty} f_{s}(\xi) d \xi
$$

Organisms whose generation times are greater than $\tau$ are those which, whatever their initial size, are of a size greater than $x_{i} e^{\nu \tau}$ at termination, since for each organism $x_{t}=x_{i} e^{\nu \tau}$. Thus

By differentiation,

$$
F_{s-}(\tau)=\int_{0}^{\infty} 2 l\left(2 x_{i}\right) L_{-}\left(x_{i} e^{\nu \tau}\right) d x_{i}
$$

$$
f_{s}(\tau)=2 \nu e^{\nu \tau} \int_{0}^{\infty} x_{i} l\left(2 x_{i}\right) l\left(x_{i} e^{\nu \tau}\right) d x_{i}
$$

in this expression it is convenient to replace $x_{i}$ by $\frac{1}{2} x_{t}$ :

$$
f_{s}(\tau)=\frac{1}{2} \nu e^{\nu \tau} \int_{0}^{\infty} x_{t} l\left(x_{t}\right) l\left(\frac{1}{2} x_{t} e^{\nu \tau}\right) d x_{t}
$$

The substitution $\xi=\sqrt{2} x_{i} e^{i \nu \tau}$, on the other hand, yields

$$
f_{s}(\tau)=\nu \int_{0}^{\infty} \xi l\left[\xi \exp \left\{-\frac{\nu}{2}\left(\tau-\frac{\log 2}{\nu}\right)\right\}\right] l\left[\xi \exp \left\{\frac{\nu}{2}\left(\tau-\frac{\log 2}{\nu}\right)\right\}\right] d \xi,
$$

which shows that $f_{s}(\tau)$ is symmetrical about $\tau=(\log 2) / \nu$, whatever the form or degree of dispersion of $x_{t}$. It follows that the arithmetic mean of $\tau$ is $(\log 2) / \nu$.

Now if we allow that the possible range of $l\left(x_{t}\right)$ is not limited, we encounter negative generation times in those instances where $x_{t}$ is less than the corresponding $x_{i}$, of which it is postulated to be independent; in fact the integral of (3) over positive $\tau$ only is less than unity. Powell's $(1955,1958)$ discussion shows that there is nothing irrational in this so far as cell-wall fission (o-fission) is concerned, and that negative generation times can very occasionally be recognized; they are awkward to deal with, however, since a generation time wrongly recorded as positive implies that another interval will be recorded which is in fact the sum of two generation times; and higher degrees of complication can conceivably occur.

We can deal with the situation in an obvious way by assuming that instances of negative generation time are negligibly few, but the assumption is uncomfortably vague until we have some means of assessing the error involved in making it. In fact, Schaechter, Williamson, Hood \& Koch (1962) noted that of the organisms they examined less than $1 \%$ contained four nuclei at any time. The progeny of such cells only could (but would not necessarily) have negative generation times. We are on firmer ground, however, if we try to find what observed pattern of growth would result from Koch \& Schaechter's postulates, the intervals between successive fissions being called (positive) generation times and labelled $\tau$; that is, if we accept the observations at their face value and disregard questions of internal structure.

According to the hypothesis, an organism has an intrinsic tendency to fission 
which at a given time depends only on its size at that time and not, in particular, on its size at inception. With this intrinsic tendency we can associate a hypothetical frequency function $\lambda\left(x_{t}\right)$ such that, if an organism has reached the size $x_{t}$, its chance of dividing before it reaches a size $x_{t}+d x_{t}$ is

$$
\lambda\left(x_{t}\right) d x_{t} / \int_{x_{t}}^{\infty} \lambda(\xi) d \xi
$$

Now if size at termination is biologically independent of size at inception, it will not in general be statistically independent of it. Just as a sexagenarian is more likely to reach the age of 70 than is a young man (other things being equal), so an organism whose size is already $x$ is more likely to attain a given size $x_{t}\left(x_{t}>x\right)$ at termination than one whose size is less than $x$. The realized and observable distribution of $x_{t}$ will not be $\lambda\left(x_{t}\right)$, but, for a given $x_{i}$, will be

where

$$
\lambda\left(x_{t}\right) / \Lambda_{-}\left(x_{i}\right),
$$

$$
\Lambda_{-}\left(x_{i}\right)=\int_{x_{i}}^{\infty} \lambda(\xi) d \xi \text {. }
$$

If an organism is of size $x_{i}$ at inception, the probability of its size at termination being greater than $x_{t}$ is

or

$$
\Lambda_{-}\left(x_{t}\right) / \Lambda_{-}\left(x_{i}\right) \quad\left(x_{t}>x_{i}\right),
$$

$$
1 \quad\left(x_{t}<x_{i}\right) .
$$

But the probability of realizing $x_{i}$ depends in the same way on the size at inception of the mother of the organism in question. We therefore introduce the realized distributions $l\left(x_{t}\right)$ of size at termination and $l_{i}\left(x_{i}\right)$ of size at inception. Then the observed frequency of fission at a size greater than $x_{t}$ will be

$$
L_{-}\left(x_{t}\right)=\int_{x_{t}}^{\infty} l(\xi) d \xi=\int_{0}^{x_{t}} \frac{\Lambda_{-}\left(x_{t}\right)}{\Lambda_{-}\left(x_{i}\right)} l_{i}\left(x_{i}\right) d x_{i}+\int_{x_{t}}^{\infty} l_{i}\left(x_{i}\right) d x_{i}
$$

the last term corresponding to the frequency with which $x_{i}$ is greater than a given $x_{t}$ (when fission necessarily occurs at a size greater than $x_{t}$ ). By differentiating twice we find in turn

$$
\begin{gathered}
\frac{l\left(x_{t}\right)}{\lambda\left(x_{t}\right)}=\int_{0}^{x_{t}} \frac{l_{i}\left(x_{i}\right)}{\Lambda_{-}\left(x_{i}\right)} d x_{i} ; \\
\frac{d}{d x_{t}}\left\{\frac{l\left(x_{t}\right)}{\lambda\left(x_{t}\right)}\right\}=\frac{l_{i}\left(x_{t}\right)}{\Lambda_{-}\left(x_{t}\right)} .
\end{gathered}
$$

We can now express $\lambda$ in terms of $l$ by treating (8) as a second-order differential equation in $\Lambda_{-}\left(x_{t}\right)$. Putting temporarily

(8) becomes

$$
W=\frac{1}{l\left(x_{t}\right)} \frac{d l\left(x_{t}\right)}{d x_{t}}, \quad V=\frac{l_{i}\left(x_{t}\right)}{l\left(x_{t}\right)}, \quad Z=\frac{\lambda\left(x_{t}\right)}{\Lambda_{-}\left(x_{t}\right)},
$$

$$
\frac{d Z}{d x_{t}}+Z^{2}(V-1)-Z W=0
$$

a Bernoulli equation soluble by conventional methods. A first integral is

$$
\frac{\lambda\left(x_{t}\right)}{\lambda\left(x_{t}\right)}=l\left(x_{t}\right) /\left\{\int_{x_{t}}^{\infty} l(\xi) d \xi-\int_{x}^{\infty} l_{i}(\xi) d \xi+A\right\},
$$


where $A$ is a constant. Now $\Lambda_{-}(0)=1$, and so the integral in (7) converges at the lower limit because $l_{i}\left(x_{i}\right)$ is a frequency function. Hence

$$
\lim _{x \rightarrow 0} \frac{l\left(x_{t}\right)}{\lambda\left(x_{t}\right)}=0
$$

Assuming for a moment that $\lambda(0)=0$, rearrangement of (9) shows that $A$ will then be zero, and

$$
\frac{\lambda\left(x_{t}\right)}{\Lambda_{-}\left(x_{t}\right)}=l\left(x_{t}\right) /\left\{L_{-}\left(x_{t}\right)-\int_{x_{t}}^{\infty} l_{i}(\xi) d \xi\right\} \text {. }
$$

The formal simplicity of this expression belies its real complexity, and an attempt to obtain an explicit formula for $\Lambda_{-}$or $\lambda$ by a further integration immediately raises difficulties of convergence. We can avoid these difficulties if we suppose that there is a non-zero lower limit to the size of an organism. This supposition is entirely acceptable biologically, and, since it also implies $\lambda(0)=0$, we can take (10) as a mathematically valid relation. It turns out that (10) as it stands is more useful than an explicit expression for $\lambda\left(x_{t}\right)$.

In particular, when organisms can be assumed to divide into daughters of equal size, we have

and (10) becomes

$$
l_{i}\left(x_{i}\right)=2 l\left(2 x_{i}\right)
$$

$$
\frac{\lambda\left(x_{t}\right)}{\Lambda_{-}\left(x_{t}\right)}=\frac{l\left(x_{t}\right)}{L_{-}\left(x_{t}\right)-L_{-}\left(2 x_{t}\right)} \text {. }
$$

\section{Fission into daughters of unequal size}

The obvious way to describe the relation between size at inception and size at termination is to introduce a bivariate distribution, $L\left(x_{t}, x_{i}\right)$, say, in which it is to be understood that $x_{i}$ is the size at inception of a daughter, $x_{t}$ the size at termination of its mother. Then the frequency function of $x_{i}$ irrespective of $x_{t}$ is

$$
\int_{x_{i}}^{\infty} \mathrm{L}\left(x_{t}, x_{i}\right) d x_{t}
$$

(since $x_{t}$ cannot be less than $x_{i}$ ), and

$$
l\left(x_{t}\right)=\int_{0}^{x_{t}} \mathrm{~L}\left(x_{t}, x_{i}\right) d x_{i} .
$$

Thus $L$ is very unsymmetrical; it can occupy only the area between the lines $x_{i}=0, x_{i}=x_{t}$ in the $x_{i}, x_{t}$ plane (Fig. $1 a$ ). Since every fission gives rise to two daughters and since, if the size of either is called $x_{i}$, the size of the other is $x_{t}-x_{i}$, the distribution of $x_{i}$ for any given $x_{t}$ is symmetrical with mean $\frac{1}{2} x_{t}$. The regression of $x_{i}$ on $x_{t}$ is $x_{i}=\frac{1}{2} x_{t}$. Suppose now we introduce a new variable $p$ :

$$
p=\frac{x_{i} \text { (daughter) }}{x_{t} \text { (mother) }}
$$

Then again the distribution of $p$ is symmetrical; $p$ and $1-p$ are equally frequent, and the possible range of $p$ is from 0 to 1 for any $x_{t}$. The joint probability element $\mathrm{L}\left(x_{t}, x_{i}\right) d x_{t} d x_{i}$ becomes $\mathrm{L}\left(x_{t}, p x_{t}\right) x_{t} d x_{t} d p$. On account of the symmetry of the $p$-distribution $\vec{p}=\frac{1}{2}$ and the ordinary product-moment correlation coefficient 
$\rho\left(x_{t}, p\right)$ is zero. But it does not follow that $p$ is independent of $x_{t}$ in any more fundamental sense. In multicellular species for example, extreme dissymmetry of fission may be relatively commoner in very long organisms where the nearly simultaneous development of several cross-walls provides opportunity for fission at a number of discrete points (Powell, 1955). Then the joint distribution of $p$ and $x_{t}$ might take some such form as Fig. $1 b$. On the other hand, in the unicellular species investigated by Schaechter et al. (1962) and Powell \& Errington (1963b) the dispersion of $p$ was very small. Accordingly Koch \& Schaechter (1962) assumed that the distribution of $p$ could be represented by a single frequency function, say $k(p)$, the same for all $x_{t}$.

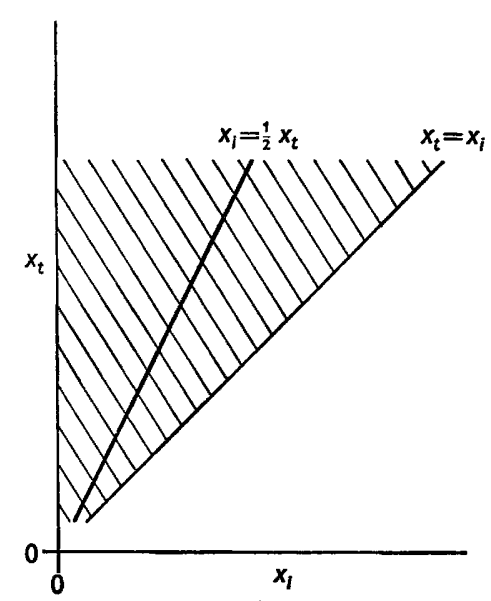

(a)

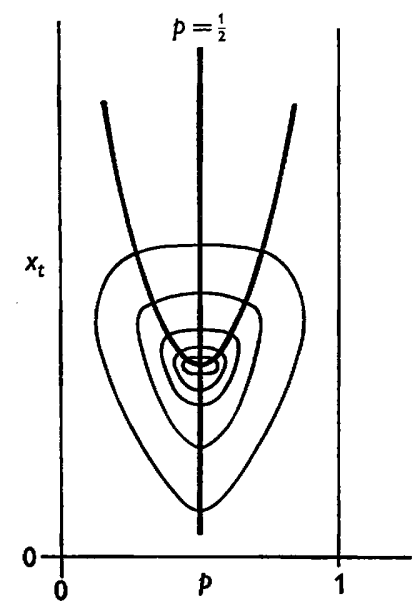

(b)

Fig. $1 a$. The joint distribution of $x_{t}$ (length at termination of mother) and $x_{i}$ (length at inception of daughter) occupies the shaded sector. The regression of $x_{i}$ on $x_{t}$ is $x_{i}=\frac{1}{2} x_{t}$. Fig. $1 b$. The joint distribution of $p\left(=x_{i} / x_{t}\right)$ and $x_{t}$ occupies the area between $p=0$ and $p=1$. The regression of $p$ on $x_{t}$ is $p=\frac{1}{2}$. The heavy parabolic line is a possible form for the regression of $x_{t}$ on $p$ which still leaves the correlation zero. The contours of the correlation surface are indicated schematically.

With this assumption, we can easily find a general expression for the distribution $l_{i}\left(x_{i}\right)$ of size at inception. The probability that an organism should be of a size greater than $x_{i}$ at inception is the probability that, whatever the size $x_{t}$ of its mother at termination, the associated value of $p$ should be greater than $x_{i} / x_{t}$, i.e.

whence

$$
\begin{aligned}
\int_{x_{i}}^{\infty} l_{i}(\xi) d \xi & =\int_{x_{i}}^{\infty} \int_{x_{i} / x t}^{1} l\left(x_{t}\right) k(p) d p d x_{t} \\
& =\int_{0}^{1} \int_{x_{i} / p}^{\infty} l\left(x_{t}\right) k(p) d x_{t} d p \\
& =\int_{0}^{1} L_{-}\left(\frac{x_{i}}{p}\right) k(p) d p,
\end{aligned}
$$

$$
l_{i}\left(x_{i}\right)=\int_{0}^{1} l\left(\frac{x_{i}}{p}\right) \frac{k(p)}{p} d p
$$


and (10) becomes

$$
\frac{\lambda\left(x_{t}\right)}{\Lambda_{-}\left(x_{t}\right)}=l\left(x_{t}\right) /\left\{L_{-}\left(x_{t}\right)-\int_{0}^{1} L_{-}\left(\frac{x_{t}}{p}\right) k(p) d p\right\} ;
$$

this agrees with (11) since, when $p$ is not dispersed, $1 / p$ takes only the value 2.

No sufficient data yet exist to show that $k(p)$ and its parameters are or are not independent of $x_{t}$, but if (12) is adopted it should be remembered that an additional assumption has been made, though perhaps not a quantitatively important one.

\section{The steady-state distribution of size}

The family tree generated by reproduction from a single organism is ideally one in which all generation times are alike equal to $(\log 2) / \nu$. Actual times of fission, because of the dispersion of size at termination, do not occur simultaneously in every generation, but, under the assumption of fission into equal daughters, are

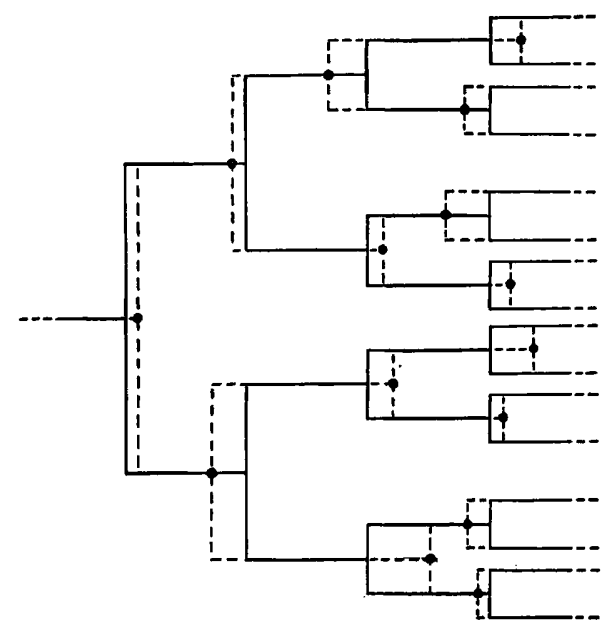

Fig. 2. The continuous line is an idealized family tree in which all generation times (horizontal segments) are alike, and all fissions in any one generation occur simultaneously. Under Koch \& Schaechter's hypothesis, actual times of fission (heavy dots) are dispersed about the ideal with a variance which is the same in every generation.

scattered about their mean with a variance $\nu^{-2} \operatorname{var} \log x_{t}$ which is the same for every generation (Fig. 2). A culture started from one organism or from a collection of organisms all of the same size would thus maintain a fixed degree of synchrony for an indefinitely long period; the number growth rate $d \log n / d t$ would be a strictly periodic function.

Among students of synchrony, the most optimistic draughtsmen know that the condition does not long persist. Qualitatively, it is easy to explain the lack of persistence. In unicellular organisms, fission usually results in sisters of nearly equal size, but there are occasional disparities which though not extravagant are detectable by simple inspection. If in a large tree such as that of Fig. 2 we imagine that a few fissions result in sisters of unequal size, the subtrees stemming from those sisters will be similar to the tree of the ancestral organism but out of phase with it. Repetition of the process will, we can suppose, lead to a culture in which all 
synchrony has been lost and the population of organisms has an unchanging size and age distribution. We have then to inquire what the steady state must be.

In view of the rarity with which pairs of markedly unequal sisters are formed, it is perhaps permissible, once the steady state has been reached, to assume that fission is symmetrical. With this restriction, Koch \& Schaechter derive a differentialdifference equation for the frequency function $\mathscr{L}\left(x_{0}\right)$ of the size of the organisms in the steady state. They also assume that, if an organism has attained a size $x$, the probability that it will divide when its size is in the range $x_{t}, x_{t}+d x_{t}$ is $\lambda\left(x_{t}\right) d x_{t} / \Lambda_{-}(x)$. That is, they make use implicitly of the assumption (5) on which we have based (11). Their equation is

$$
\frac{d}{d x_{0}}\left\{x_{0}^{2} \mathscr{L}\left(x_{0}\right)\right\}=2\left(2 x_{0}\right)^{2} \mathscr{L}\left(2 x_{0}\right) \frac{\lambda\left(2 x_{0}\right)}{\Lambda_{-}\left(2 x_{0}\right)}-x_{0}^{2} \mathscr{L}\left(x_{0}\right) \frac{\lambda\left(x_{0}\right)}{\Lambda_{-}\left(x_{0}\right)} .
$$

Koch \& Schaechter's reasoning so far seems to be impeccable, but they go on to suggest that

$$
\mathscr{L}\left(x_{0}\right)=\frac{C}{x_{0}^{2}} \int_{x_{0}}^{2 x_{0}} \lambda(\xi) d \xi \quad(C \text { a constant })
$$

is an approximate solution of (14). It is difficult to subscribe to the considerations by which they arrive at (15).

It is easy to verify by substitution that an exact solution of (14) is

$$
\mathscr{L}\left(x_{0}\right)=\frac{C}{x_{0}^{2}} \int_{x_{0}}^{2 x_{0}} l(\xi) d \xi=\frac{C}{x_{0}^{2}}\left\{L_{-}\left(x_{0}\right)-L_{-}\left(2 x_{0}\right)\right\},
$$

where $C$ is a constant and the relation between $l$ and $\lambda$ is given by (11). Since $\mathscr{L}$ is a frequency function

$$
\begin{aligned}
1 & =\int_{0}^{\infty} \mathscr{L}\left(x_{0}\right) d x_{0} \\
& =C\left[\frac{L_{-}\left(x_{0}\right)-L_{-}\left(2 x_{0}\right)}{x_{0}}\right]_{0}^{\infty}+C \int_{0}^{\infty} \frac{2 l\left(2 x_{0}\right)-l\left(x_{0}\right)}{x_{0}} d x_{0}
\end{aligned}
$$

on integrating by parts. It is found by differentiation that the first term on the right is zero at the lower limit, and so

$$
\begin{aligned}
1 & =2 C \int_{0}^{\infty} \frac{l\left(2 x_{0}\right)}{2 x_{0}} d\left(2 x_{0}\right)-C \int_{0}^{\infty} \frac{l\left(x_{0}\right)}{x_{0}} d x_{0} \\
& =C \int_{0}^{\infty} \frac{l\left(x_{0}\right)}{x_{0}} d x_{0} .
\end{aligned}
$$

The last integral is the moment of order -1 of $l\left(x_{0}\right)$; thus $C$ is the harmonic mean of the distribution $l$.

The further extension to unsymmetrical fission is less simple. Koch \& Schaechter's analysis can be carried through as before, but the contribution to $d\left\{x_{0}^{2} \mathscr{L}\left(x_{0}\right)\right\} / d x_{0}$ by newly formed organisms does not now come only from organisms with $x_{t}=2 x_{0}$, but from those whose size at termination is $x_{0} / p$; and $p$ takes any value in the range 0 to 1 with frequency $k(p)$. Equation (14) now becomes

$$
\frac{d}{d x_{0}}\left\{x_{0}^{2} \mathscr{L}\left(x_{0}\right)\right\}=2 \int_{0}^{1}\left(\frac{x_{0}}{p}\right)^{2} \mathscr{L}\left(\frac{x_{0}}{p}\right) \frac{\lambda\left(x_{0} / p\right)}{\Lambda_{-}\left(x_{0} / p\right)} k(p) d p-x_{0}^{2} \mathscr{L}\left(x_{0}\right) \frac{\lambda\left(x_{0}\right)}{\Lambda_{-}\left(x_{0}\right)} .
$$


If we take

$$
x_{0}^{2} \mathscr{L}\left(x_{0}\right)=L_{-}\left(x_{0}\right)-\int_{0}^{1} k(p) L_{-}\left(x_{0} / p\right) d p
$$

as a trial solution of (18) (by analogy with (16)) we obtain after reduction

$$
\int_{0}^{1} \frac{k(p)}{p} l\left(\frac{x_{0}}{p}\right) d p=2 \int_{0}^{1} k(p) l\left(\frac{x_{0}}{p}\right) d p,
$$

which is in general false. The two integrals have nearly the same value, since $p$ rarely differs much from $\frac{1}{2}$, but (19) is not exact. An exact solution of (18) is derived from Collins \& Richmond's (1962) analysis in the next section but one.

\section{Real and artificial distributions}

It is convenient at this point to introduce an excursus on the relation between conceptual distributions of generation time or size and the distributions naturally presented by a steadily growing culture. The considerations which I now advance have not, I think, been made explicit elsewhere in the bacteriological literature; they also constitute a justification for the multiplicity of distribution functions which have to be handled in discussing the pattern of growth.

The idea of a distribution of generation times $f(\tau)$ is so familiar as to seem simple. It is not so. Suppose first that generation times are independent of one another, as in Kendall's or Rahn's hypothesis. We can obtain a sample of $\tau$ by fixing our attention on a group of fissions selected in any way and recording the $\tau$ of all the organisms stemming immediately from those fissions. Repetition of the process generates the frequency function $f(\tau)$. But the generation times of the organisms which have, at a given time, completed their life span during the previous history of the culture do not compose $f(\tau)$; they compose the carrier distribution $\mathscr{C}(\tau)$ (Powell, 1956):

$$
\mathscr{C}(\tau) \equiv 2 e^{-\nu \tau} f(\tau)
$$

The distribution of $\tau$ among those organisms extant at a given time is, say, $\mathscr{I}(\tau)$, and

$$
\mathscr{I}(\tau) \equiv 2\left(1-e^{-\nu \tau}\right) f(\tau) .
$$

(I previously stated, incorrectly (Powell, 1956), that $\mathscr{I}(\tau) \equiv f(\tau)$.) When generation times are not independent of one another (as they are not in the hypothesis of Koch \& Schaechter and in fact), it is more difficult to obtain an unbiased sample of the $\tau$. For suppose that the generation time of an organism depends on that of its mother. The number of fissions, occurring at any given time, which are the terminations of organisms of generation time $\tau$ is proportional to $e^{-\nu \tau}$; at the inception of these organisms the number in the culture, and therefore the absolute fission rate, was less by this factor. (In a steady state continuous culture terminations of organisms of large $\tau$ are similarly rare, because the probability of the organisms' remaining in the vessel until termination is also $e^{-\nu \tau}$.) It is not enough, then, to study the organisms stemming from fissions selected apparently at random, because the $\tau$ thus made available will be influenced by the $\tau$ of the mothers. We may obtain a representative sample by agreeing in advance to record the $\tau$ of all the $n$th generation progeny of selected organisms, $n$ being large enough for these $\tau$ to be uninfluenced by the original selection. (In practice, $n=2$ is probably sufficient to secure a close approximation to independence.) The carrier distribution is no longer 
given by (20); organisms whose $\tau$ belongs to it must be such that their progeny, after allowance for increase in numbers (or for washout, in continuous cultures) themselves regenerate the carrier distribution. We imagine a 'population' distribution $\mathscr{P}(\tau)$ such that

$$
\mathscr{C}(\tau) \equiv 2 e^{-\nu \tau} \mathscr{P}(\tau)
$$

Then $\mathscr{P}(\tau)$ is given in terms of $f(\tau)$ and the joint distribution of mother and daughter generation times by means of an integral equation (Powell, 1956). The culture behaves as if the generation times were independent of one another and had the distribution $\mathscr{P}(\tau) ; \mathscr{P}(\tau)$ replaces $f(\tau)$ in expressions for the carrier and age distributions and for $\mathscr{I}(\tau)$ (equation(21)). The relation between $\mathscr{C}(\tau), \mathscr{P}(\tau)$ and $f(\tau)$ is perhaps most easily seen by considering an imaginary continuous culture with a steady flow of liquid through it. Suppose that, every time a fission occurred, one of the new organisms was selected at random (i.e. without reference to its then unknown generation time) and allowed to leave the culture. The organisms in the vessel would remain constant in number and their generation time distribution would be $f(\tau)$. In a real culture, removal from the vessel depends upon generation time. Those organisms which remain to complete their life span in the vessel have the distribution $\mathscr{C}(\tau)$ : those which so remain, together with those which are washed out before termination, have the distribution $\mathscr{P}(\tau)$.

In Koch \& Schaechter's hypothesis, $l\left(x_{t}\right)$ corresponds to $f(\tau)$, and we have now to introduce distributions, say $l_{o}\left(x_{t}\right)$ and $l_{P}\left(x_{t}\right)$ corresponding to $\mathscr{C}(\tau)$ and $\mathscr{P}(\tau)$. For example, those organisms whose generation times compose $\mathscr{C}(\tau)$ are those whose sizes at termination compose $l_{o}\left(x_{t}\right)$.

\section{Collins \& Richmond's analysis}

Collins \& Richmond (1962) derived an equation connecting the mean rate of growth of individual organisms $\left(V_{x}\right)$ with the distributions of size at inception and termination and the instantaneous distribution of size at any one time. In its original form it reads

$$
V x=k\left\{2 \int_{0}^{l_{x}} \Psi(l) d l-\int_{0}^{l_{x}} \Phi(l) d l-\int_{0}^{l_{x}} \lambda(l) d l\right\} / \lambda\left(l_{x}\right) .
$$

Collins \& Richmond's $\lambda$ is our $\mathscr{L}$, their $k$ is our $\nu$, their $l_{x}$ is our $x_{0}$ and, in Koch \& Schaechter's hypothesis, $V x$ is simply $\nu x$, since $d \log x / d t=\nu$. We can write, retaining $\Psi$ and $\Phi$ for the moment,

$$
x_{0} \mathscr{L}\left(x_{0}\right)=2 \int_{0}^{x_{0}} \Psi(\xi) d \xi-\int_{0}^{x_{0}} \Phi(\xi) d \xi-\int_{0}^{x_{0}} \mathscr{L}(\xi) d \xi .
$$

On differentiating, we obtain a differential equation easily soluble for $\mathscr{L}$ :

and

$$
x_{0} \frac{d \mathscr{L}}{d x_{0}}+2 \mathscr{L}=2 \Psi-\Phi
$$

If fission is symmetrical,

$$
\mathscr{L}\left(x_{0}\right)=\frac{1}{x_{0}^{2}} \int_{0}^{x_{0}} \xi\{2 \Psi(\xi)-\Phi(\xi)\} d \xi .
$$

$$
\begin{aligned}
\mathscr{L}\left(x_{0}\right) & =\frac{1}{x_{0}^{2}} \int_{0}^{x_{0}} \xi\{4 \Phi(2 \xi)-\Phi(\xi)\} d \xi \\
& =\frac{1}{x_{0}^{2}} \int_{x_{0}}^{2 x_{0}} \xi \Phi(\xi) d \xi .
\end{aligned}
$$


Collins \& Richmond's distribution of size at termination, $\Phi\left(x_{t}\right)$, is not our $l\left(x_{t}\right)$; it is the distribution $l_{C}\left(x_{t}\right)$ of the size at termination of those organisms whose $\tau$ belong to $\mathscr{C}(\tau)$ (compare (24) with (16)). It follows that when fission is symmetrical

$$
l_{C}\left(x_{t}\right)=C l\left(x_{t}\right) / x_{t} \text {. }
$$

More generally when fission is not symmetrical, the distribution of size at inception actually realized up to a given time in a growing culture is

and (22) becomes

$$
\int_{0}^{1} \frac{k(p)}{p} l_{c}\left(\frac{x_{i}}{p}\right) d p
$$

$$
\mathscr{L}\left(x_{0}\right)=\frac{1}{x_{0}^{2}}\left\{2 \int_{0}^{x_{0}} \int_{0}^{1} \xi l_{c}\left(\frac{\xi}{p}\right) \frac{k(p)}{p} d p d \xi-\int_{0}^{x_{0}} \xi l_{C}(\xi) d \xi\right\}
$$

We can obtain still another relation between $\mathscr{L}\left(x_{0}\right)$ and $l_{c}\left(x_{t}\right)$. When the number in the culture is $N$, there are $N \mathscr{L}\left(x_{0}\right) d x_{0}$ organisms whose size is in the range $x_{0}$, $x_{0}+d x_{0}$. During a short time $d t$ there are $N \nu d t$ fissions which are the termination of organisms belonging to $l_{o}\left(x_{t}\right)$. In the same interval organisms of size $x_{0}$ would grow, in the absence of fission, from $x_{0}$ to $x_{0} e^{\nu d t}, \bumpeq x_{0}(1+\nu d t)$-an increment of $x_{0} \nu d t$. The number which divide is thus

$$
N \mathscr{L}\left(x_{0}\right) d x_{0} \frac{\lambda\left(x_{0}\right)}{\Lambda_{-}\left(x_{0}\right)} x_{0} \nu d t
$$

But the number dividing in the size range $x_{0}, x_{0}+d x_{0}$ is also $N l_{c}\left(x_{0}\right) d x_{0} \nu d t$ and so

$$
l_{c}\left(x_{t}\right)=\mathscr{L}\left(x_{t}\right) \frac{x_{t} \lambda\left(x_{t}\right)}{\Lambda_{-}\left(x_{t}\right)} .
$$

It is easily found by substitution that (25) and (26) are together consistent with the general form of Koch \& Schaechter's equation (18). Also they give a relation between $\lambda$ and $l_{c}$ analogous to (13):

$$
\frac{\lambda\left(x_{t}\right)}{\Lambda_{-}\left(x_{t}\right)}=x_{t} l_{c}\left(x_{t}\right) /\left\{2 \int_{0}^{x_{t}} \int_{0}^{1} \xi \frac{k(p)}{p} l_{\sigma}\left(\frac{\xi}{p}\right) d p d \xi-\int_{0}^{x_{t}} \xi l_{C}(\xi) d \xi\right\} .
$$

\section{Approximate distributions of size and generation time}

It is now easy to elaborate a series of varied and complex relations between $\lambda, l, l_{C}, l_{P}, \mathscr{L}, f, \mathscr{C}$ and $\mathscr{P}$. Most of them are beyond experimental test for want of data sufficiently numerous and precise. Moreover, no hypothesis about the pattern of growth is likely to agree more than broadly with the known phenomena. It is therefore useless to pursue mathematical exactitude; instead we should seek some approximation which will make algebraic manipulation simpler and such that its magnitude can be estimated experimentally.

\section{A simplifying approximation}

Let us suppose that every organism passes through a size $X$ at some time during its life span. This implies that the two distributions $l_{i}, l$ do not overlap; i.e. the largest $x_{i}$ ever to occur is not greater than the smallest $x_{t}: X$ lies between these extremes. (It will be convenient in the sequel to take $X$ to be equal to or just less than the smallest $x_{t}$.) It implies further a restriction on the range of $p: p x_{t}$ must never be greater than $X$. 
On the assumption that the distribution of $p$ is independent of $x_{t}$ we can suppose its range to be say $\frac{1}{2} \pm \epsilon$. Then the range of $x_{t}$ must be from $X$ to $X /\left(\frac{1}{2}+\epsilon\right)$ and of $x_{i}$ from $X\left(\frac{1}{2}-\epsilon\right)$ to $X$. The range of the joint distribution of $x_{i}$ and $x_{t}$ is illustrated in Fig. 3. The corresponding range of generation times will be from $\tau=0$ (when

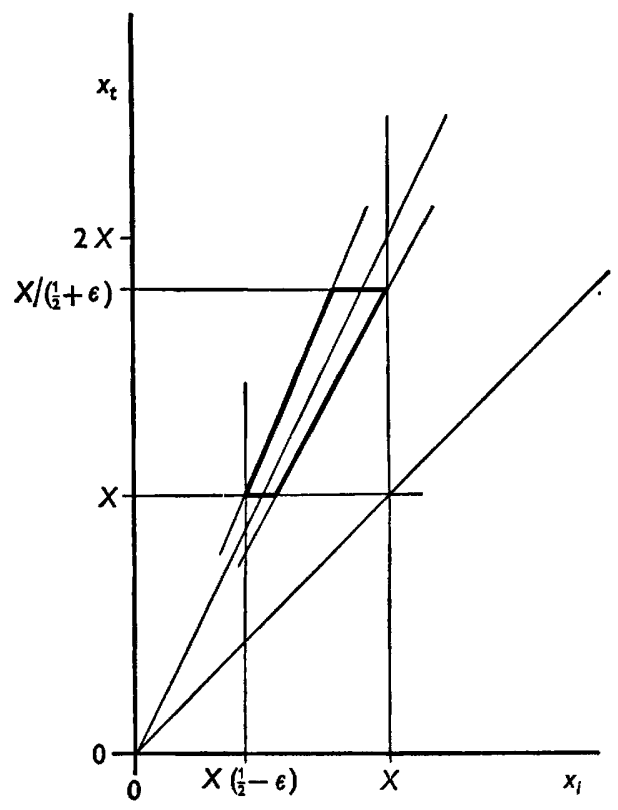

Fig. 8. When the distributions of $x_{t}$ and $x_{t}$ do not overlap, the joint distribution is confined to the interior of a trapezium (heavy line). Its limits are given in terms of the critical size $X$ and the range $\frac{1}{2} \pm \epsilon$ of the ratio $p$.

for the same organism $x_{i}=X=x_{t}$ ) to a little more than $2 \bar{\tau}$ (when $x_{i}=X\left(\frac{1}{2}-\epsilon\right)$ and $x_{t}=X /\left(\frac{1}{2}+\epsilon\right)$, and $\left.\tau=\log \left(x_{t} / x_{i}\right) / \nu \bumpeq 2 \bar{\tau}\right)$. In particular, $l_{i}\left(x_{i}\right)$ will be zero for $x_{i}>X$ and so in equations (10) and (13) the second term in the denominator on the right will vanish for all $x_{t}$ for which $l\left(x_{t}\right)$ is not 0 .

Under this simplifying assumption, then, we have

$$
\lambda\left(x_{t}\right) \equiv l\left(x_{t}\right)
$$

that is, the realized distribution of length at fission is identical with Koch \& Schaechter's intrinsic distribution $\lambda\left(x_{t}\right)$. Koch \& Schaechter based their treatment on the assumption that the coefficient of variation of $x_{t}$ was small; in fact it is the limited range which is the necessary condition for the truth of their inferences; the only logical restriction on the coefficient of variation is then that it should be less than $1 / \sqrt{8} \bumpeq 0.35$.

The distribution of generation times

Under the simplifying assumption we have just made, equation (3) represents the distribution of $\tau$ when fission results in daughters of equal size. More generally, the factor $2 l\left(2 x_{i}\right)$ in (2) has to be replaced by (12), and

$$
f(\tau)=\nu \int_{0}^{\infty} \int_{0}^{1} x_{i} e^{\nu \tau} \frac{k(p)}{p} l\left(\frac{x_{i}}{p}\right) l\left(x_{i} e^{\nu \tau}\right) d p d x_{i},
$$


where the subscript $s$ of $f$ has been dropped since this frequency function is not symmetrical. It has now to be remembered that the integrand in (29) is zero when the argument of either of the $l$-functions lies outside the range $X, 2 X$; the effective limits of the integral (29) are

$$
x_{i}\left(\frac{1}{2}+\varepsilon\right) / X<p<x_{i} \mid X \text { and } X e^{-\nu \tau}<x_{i}<X e^{-v \tau} /\left(\frac{1}{2}+\varepsilon\right) .
$$

The mean of the distribution is

$$
\tau=\nu \iiint \tau e^{\nu \tau} \frac{k(p)}{p} l\left(\frac{x_{i}}{p}\right) l\left(x_{i} e^{\nu \tau}\right) x_{i} d p d x_{i} d \tau
$$

the integration being carried over the whole range for which $l$ and $k$ are not 0 . On putting $x_{t}=x_{i} / p$ and $\xi=x_{i} e^{\nu \tau}$ it becomes

$$
\begin{aligned}
\bar{\tau} & =\frac{1}{\nu} \iiint\left(\log \xi-\log x_{t}-\log p\right) k(p) l\left(x_{t}\right) l(\xi) d p d x_{t} d \xi \\
& =-\frac{1}{\nu} \int(\log p) k(p) d p \\
& =-\mu_{1}^{\prime}(\log p) / \nu .
\end{aligned}
$$

The geometric mean of $p$, namely $\exp \left\{\mu_{1}^{\prime}(\log p)\right\}$, is by a general rule less than its arithmetic mean $\left(\frac{1}{2}\right)$, hence $-\mu_{1}^{\prime}(\log p)>\log 2$ : for a given $v, \bar{\tau}$ is increased by the fission of organisms into daughters of unequal size.

The other moments about the origin are given by

$$
\mu_{r}^{\prime}(\tau)=\nu \iiint \tau^{r} e^{\nu \tau} \frac{k(p)}{p} l\left(\frac{x_{i}}{p}\right) l\left(x_{i} e^{\nu \tau}\right) x_{i} d p d x_{i} d \tau
$$

The same substitution as before yields

$$
\mu_{r}^{\prime}(\tau)=\nu^{-r} \iiint\left(\log \xi-\log x_{t}-\log p\right)^{r} k(p) l\left(x_{t}\right) l(\xi) d p d x_{t} d \xi
$$

This expression can be dealt with expeditiously by noting that the right-hand side is the $r$ th derivative with respect to $s$ of

$$
\iiint\left(\xi / p x_{t}\right)^{(s-1) / \nu} k(p) l\left(x_{t}\right) l(\xi) d p d x_{t} d \xi
$$

when $s=1$. The factor $\left(\xi / p x_{t}\right)^{(s-1) / \nu}$ can be written

and so

$$
\xi^{(s+\nu-1) / \nu-1} \cdot p^{(\nu+1-8) / \nu-1} \cdot x_{t}^{(\nu+1-s) / \nu-1},
$$

$$
\left.\mu_{r}^{\prime}(\tau)=\frac{d^{r}}{d s^{r}}\left\{\mathfrak{M}\left(\frac{\nu+s-1}{\nu} ; l\right) \mathfrak{M}\left(\frac{\nu+1-s}{\nu} ; l\right) \mathfrak{M}\left(\frac{\nu+1-s}{\nu}\right) ; k\right)\right\}\left.\right|_{s=1},
$$

where $\mathfrak{M}(s ; l), \mathfrak{M}(s ; k)$ are respectively the Mellin transforms of $l\left(x_{t}\right)$ and $k(p)$. Since the operand in (31) generates the moments, its logarithm generates the cumulants :

$$
\kappa_{r}(\tau)=\left.\frac{d^{r}}{d s^{r}}\left[\log \left\{\mathfrak{M}\left(\frac{\nu+s-1}{\nu} ; l\right) \mathfrak{M}\left(\frac{\nu+1-s}{\nu} ; l\right) \mathfrak{M}\left(\frac{\nu+1-s}{\nu} ; k\right)\right\}\right]\right|_{s=1} .
$$

In particular

$$
\operatorname{var} \tau=\kappa_{2}(\tau)=\nu^{-2}\left(2 \operatorname{var} \log x_{t}+\operatorname{var} \log p\right),
$$

or writing $c$ for a coefficient of variation, and putting $\nu=-\mu_{1}^{\prime}(\log p) / \bar{\tau}$,

$$
c^{2}(\tau) \bumpeq\left\{2 c^{2}\left(x_{t}\right)+c^{2}(p)\right\} /\left\{\mu_{1}^{\prime}(\log p)\right\}^{2} .
$$


When $p$ is not appreciably dispersed,

so

$$
\begin{gathered}
c^{2}(p) \ll c^{2}\left(x_{t}\right) \quad \text { and } \mu_{1}^{\prime}(\log p) \bumpeq \log \frac{1}{2} ; \quad\left(\log \frac{1}{2}\right)^{2} \bumpeq \frac{1}{2}, \\
c^{2}(\tau) \bumpeq 4 c^{2}\left(x_{t}\right) .
\end{gathered}
$$

Thus $c(\tau)$ is about twice $c\left(x_{t}\right)$. Schaechter et al. (1962) found this to be roughly true of the organisms they examined.

The third moment of $f(\tau)$, from (32), is

$$
\mu_{3}(\tau)=\kappa_{3}(\tau)=-\mu_{3}(\log p) / \nu^{3},
$$

and from this we find that the skewness of $f(\tau)$ is

$$
\gamma_{1}(\tau)=\mu_{3}(\tau) /\left\{\mu_{2}(\tau)\right\}^{\frac{3}{2}}=\frac{-\mu_{3}(\log p)}{\left\{-\mu_{1}^{\prime}(\log p)\right\}^{3} c^{3}(\tau)}
$$

this is a positive number since the expectations of odd powers of $\log p$ are all negative. As Koch \& Schaechter suggested, lack of symmetry at fission introduces positive skewness into $f(\tau)$.

The application of an integral transform gives us a general method of handling (29), particularly when specific frequency functions are involved, but the simple results (30) and (33) can be obtained more readily by a direct appeal to the equation

$$
\tau=\frac{1}{v} \log \left(\frac{x_{i}^{\prime}}{x_{i}}\right),
$$

in which it is to be understood that $x_{i}$ and $x_{i}^{\prime}$ belong to the same organism, not the same fission.

\section{The carrier distributions}

The simplifying assumption implies that the size of an organism at termination is statistically as well as biologically independent of its size at inception. Since each organism passes through the size $X$, its contributions to $l_{O}\left(x_{t}\right), l_{P}\left(x_{t}\right), l\left(x_{t}\right)$ can only differ because of its history after reaching $X$. We can say at once that $l_{C}\left(x_{t}\right)$ is proportional to $l\left(x_{t}\right) e^{-v} t_{X}$, where $t_{X}$ is the time that elapses between the attainment of size $X$ and termination. But by the original postulates

and so

$$
x_{t}=X e^{\nu t}
$$

$$
l_{c}\left(x_{t}\right) \propto l\left(x_{t}\right) / x_{t} .
$$

The constant of proportionality is evidently the harmonic mean, $C$, of the distribution $l\left(x_{t}\right)$ (equation 17). This accords with (27); obviously $l_{c}$ and $l$ have the same range, and the first integral in the denominator of (27) covers the whole range of $l_{c}(\xi / p)$ for any $x_{t}$ for which $l_{C}\left(x_{t}\right)$ is not zero. This term becomes

$$
\begin{aligned}
2 \int_{0}^{1} \int_{0}^{x t} p k(p) \frac{\xi}{p} l_{C}\left(\frac{\xi}{p}\right) \frac{d \xi}{p} d p & =2 C \int_{0}^{1} p k(p) d p \\
& =C ; \\
C-\int_{0}^{x t} \xi l_{C}(\xi) d \xi & =\int_{x t}^{\infty} \xi l_{C}(\xi) d \xi
\end{aligned}
$$

and

$$
\frac{x_{t} l_{o}\left(x_{t}\right)}{\int_{x_{t}}^{\infty} \xi l_{o}(\xi) d \xi}=\frac{\lambda\left(x_{t}\right)}{\Lambda_{-}\left(x_{t}\right)}=\frac{l\left(x_{t}\right)}{L_{-}\left(x_{t}\right)} \text {. }
$$


Thus we now have, whatever the dispersion of $p$,

$$
l_{c}\left(x_{t}\right) \equiv C l\left(x_{t}\right) / x_{t}
$$

The carrier distribution of generation time, $\mathscr{C}(\tau)$, can now be written down at once, by comparison with (29):

$$
\begin{aligned}
\mathscr{C}(\tau) & =\nu \int_{0}^{\infty} \int_{0}^{1} \frac{k(p)}{p} l_{C}\left(\frac{x_{i}}{p}\right) l_{C}\left(x_{i} e^{\nu \tau}\right) x_{i} e^{\nu \tau} d p d x_{i} \\
& =C^{2} \nu \int_{0}^{\infty} \int_{0}^{1} \frac{k(p)}{x_{i}} l\left(\frac{x_{i}}{p}\right) l\left(x_{i} e^{\nu \tau}\right) d p d x_{i} .
\end{aligned}
$$

It is clear that under the simplifying assumption $l_{P}\left(x_{t}\right)$, corresponding to $\mathscr{P}(\tau)$, is identical with $l\left(x_{t}\right)$, because now each $x_{t}$ is independent of the preceding $x_{i}$. But it is not true that $\mathscr{P}(\tau) \equiv f(\tau)$ because the $\tau$ depend on the $x_{i}$ as well as the $x_{t}$. A special investigation is not necessary; since

we have

$$
2 e^{-\nu \tau} \mathscr{P}(\tau) \equiv \mathscr{C}(\tau),
$$

$$
\mathscr{P}(\tau)=\frac{C^{2} \nu e^{\nu \tau}}{2} \int_{0}^{\infty} \int_{0}^{1} \frac{k(p)}{x_{i}} l\left(\frac{x_{i}}{p}\right) l\left(x_{i} e^{\nu \tau}\right) d p d x_{i} .
$$

The frequency function of age. $\phi(a)$, is given by

$$
\phi(a)=2 v e^{-\nu a} \int_{a}^{\infty} \mathscr{P}(\tau) d \tau
$$

on replacing $\mathscr{P}(\tau)$ from (35) and changing the order of integration, it becomes

$$
\phi(a)=C^{2} \nu e^{-\nu a} \int_{0}^{\infty} \int_{0}^{1} \frac{k(p)}{x_{i}} l\left(\frac{x_{i}}{p}\right) L_{-}\left(x_{i} e^{\nu a}\right) d p d x_{i} .
$$

\section{Correlations between generation times}

The principal correlations which have to be accounted for by any hypothesis are those between the generation times of mothers and daughters, and between the generation times of sisters.

Suppose we have an organism of generation time $\tau_{m}$ whose extremes of size are $x^{\prime}, x_{t}^{\prime}$. Let the generation time of one of its daughters be $\tau_{d}$ and let the extreme sizes of this daughter be $x_{i}, x_{t}$. Then $x_{i}$ is a fraction $p$ of $x_{i}^{\prime}$ and

i.e.

$$
x_{t}=x_{i} \exp \left\{\nu \tau_{d}\right\}=p x_{t}^{\prime} \exp \left\{\nu \tau_{d}\right\}=p x_{i}^{\prime} \exp \left\{\nu\left(\tau_{d}+\tau_{m}\right)\right\}
$$

Then

$$
\tau_{d}+\tau_{m}=\frac{1}{\nu} \log \left(\frac{x_{t}}{x_{i}^{\prime} p}\right)
$$

$$
\operatorname{var}\left(\tau_{d}+\tau_{m}\right)=\nu^{-2}\left(\operatorname{var} \log x_{t}+\operatorname{var} \log x_{i}^{\prime}+\operatorname{var} \log p\right) ;
$$

under the assumptions that $p$ is independent of the $x_{t}$ with which it is associated and that the distributions $l\left(x_{t}\right), l_{i}\left(x_{i}\right)$ do not overlap, the covariances between $x_{t}, x_{i}^{\prime}$ and $p$ are all zero. We have now to relate var $\log x_{i}^{\prime}$ to var $\log x_{t}$; since these statistics are characteristics of functions $l_{i}\left(x_{i}\right), l\left(x_{t}\right)$, the prime is irrelevant. Every $x_{i}$ belongs to the same fission as some $x_{t}$, and then

Hence

$$
\begin{gathered}
x_{i}=p x_{t} ; \\
\operatorname{var} \log x_{i}=\operatorname{var} \log p+\operatorname{var} \log x_{t} .
\end{gathered}
$$

$$
\operatorname{var}\left(\tau_{d}+\tau_{m}\right)=2 \nu^{-2}\left(\operatorname{var} \log x_{t}+\operatorname{var} \log p\right)
$$


But $\rho(H)$, the product-moment correlation between the generation times of mothers and daughters, is given by

and we find, using (33),

$$
\rho(H)=\frac{\operatorname{var}\left(\tau_{d}+\tau_{m}\right)}{2 \operatorname{var} \tau}-1,
$$

$$
\rho(H)=\frac{-\operatorname{var} \log x_{t}}{2 \operatorname{var} \log x_{t}+\operatorname{var} \log p}
$$

The corresponding analysis for sisters is a little more complicated. If $\tau_{1}, \tau_{2}$ are the generation times of a pair of sisters whose terminal sizes are $x_{t}^{\prime}, x_{t}^{\prime \prime}$, we have

$$
\tau_{1}=\frac{1}{\nu} \log \left(\frac{x_{i}^{\prime}}{p x_{t}}\right) ; \quad \tau_{2}=\frac{1}{\nu} \log \left\{\frac{x_{i}^{\prime \prime}}{(1-p) x_{t}}\right\}
$$

where $x_{t}$ is the terminal size of the mother. Then

$$
\begin{aligned}
\tau_{1}-\tau_{2} & =\frac{1}{\nu} \log \left\{\frac{x_{t}^{\prime}(1-p)}{p x_{t}^{\prime \prime}}\right\} ; \\
\operatorname{var}\left(\tau_{1}-\tau_{2}\right) & =\nu^{-2}\left[2 \operatorname{var} \log x_{t}+\operatorname{var} \log \{(1-p) / p\}\right] .
\end{aligned}
$$

By symmetry, $\operatorname{var} \log (1-p)=\operatorname{var} \log p$, but here $p$ and $1-p$ arealgebraically related, not separate realizations of the variate $p$. Writing $E$ for expectation, we have

also

$$
\begin{aligned}
\operatorname{var} \log p & =E(\log p)^{2}-\{E(\log p)\}^{2} \\
\operatorname{var} \log \{(1-p) / p\} & =2 E(\log p)^{2}-2 E\{\log p \cdot \log (1-p)\} ;
\end{aligned}
$$

So

$$
\operatorname{var} \log \{p(1-p)\}=2 E(\log p)^{2}+2 E\{\log p \log (1-p)\}-4\{E(\log p)\}^{2} \text {. }
$$

$$
\begin{aligned}
\operatorname{var} \log \{(1-p) / p\}+\operatorname{var} \log \{p(1-p)\} & =4 E(\log p)^{2}-4\{E(\log p)\}^{2} \\
& =4 \operatorname{var} \log p,
\end{aligned}
$$

and

$$
\operatorname{var}\left(\tau_{1}-\tau_{2}\right)=\nu^{-2}\left[2 \operatorname{var} \log x_{t}+4 \operatorname{var} \log p-\operatorname{var} \log \{p(1-p)\}\right] .
$$

The expression $p(1-p)$, qua function of $p$, has a maximum at $p=\frac{1}{2}$; it therefore changes little over the important range: the observed $p$ are closely grouped about $p=\frac{1}{2}$. Thus var $\log \{p(1-p)\}$ is relatively small. If we omit this term, we have, very nearly,

$$
\begin{aligned}
\rho(S) & =1-\frac{\operatorname{var}\left(\tau_{1}-\tau_{2}\right)}{2 \operatorname{var} \tau} \\
& =\frac{\operatorname{var} \log x_{t}-\operatorname{var} \log p}{2 \operatorname{var} \log x_{t}+\operatorname{var} \log p} .
\end{aligned}
$$

Koch \& Schaechter's approximate formulae, in which coefficients of variation replace variances of logarithms, agree with (36) and (37).

When the dispersion of $p$ is small enough, then,

$$
-\rho(H)=\rho(S)=\frac{1}{2} \text {. }
$$

Koch \& Schaechter's hypothesis is an advance on all previous ideas in that it predicts that $\rho(H)$ and $\rho(S)$ will not be negligible and will be respectively negative and positive. It does not account for the deeper hereditary properties evinced as correlations between the $\tau$ of cousins and second cousins, nor does it account 
quantitatively for the divergence of $-\rho(H), \rho(S)$ from $\frac{1}{2}$. According to (36) and (37), the divergences result from the dispersion of $p$, and

$$
\begin{gathered}
\rho(S) \leqslant-\rho(H) \leqslant \frac{1}{2} ; \\
\rho(S)+\rho(H) \leqslant 0 .
\end{gathered}
$$

In practice the inequalities are not found to be true; with every allowance for experimental imperfection, $\rho(S)$ usually exceeds $\frac{1}{2}$ and $-\rho(H)$ rarely attains it (Powell, 1958; Powell \& Errington, 1963a; Schaechter et al. 1962).

\section{Justification of the assumptions}

It is not the purpose of this note to attempt a general critique of the Koch \& Schaechter hypothesis, but only to suggest how, by a mathematical simplification, its consequences can best be compared with experiment. We arrive at manageable formulae by making two special assumptions, but we cannot fairly use these formulae to test the hypothesis unless the assumptions which make them valid are true (or very nearly true) of the data to which they are to be applied. Accordingly I now adduce some evidence that the assumptions are often true of real cultures.

The first and probably much the less important assumption is that inequality in the size of sister organisms at inception is independent of the size of the mother at termination; more precisely, that the distribution of the parameter $p$ is the same for all $x_{t}$. There is as yet no direct evidence bearing on this point.

The second assumption is that the distributions of length at inception and length at termination do not overlap or, what is logically equivalent, that there is a size $\boldsymbol{X}$ which is attained by every organism during its life span. A further implication is that $p$ and $x_{t}$ are jointly restricted so that $p x_{t} \leqslant X$ for all $p$, and we can suppose that the range of $p$ is $\frac{1}{2} \pm \epsilon$. It now follows that the possible extremes of $x_{i}$ are $X$, $X\left(\frac{1}{2}-\epsilon\right)$-a range of a little more than $2: 1$; for $x_{t}$ they are $X, X /\left(\frac{1}{2}+\epsilon\right)$-a range of a little less than 2:1. Further, the possible extremes of $x_{0}$, the cell size in the steadystate population, are $X\left(\frac{1}{2}-\epsilon\right), X /\left(\frac{1}{2}+\epsilon\right)$-a range of a little more than $4: 1$.

The measurements of Collins \& Richmond (1962) on Bacillus cereus provide an interesting example. Within the accuracy of their technique, they found the ratio of the extremes of $x_{0}$ to be just $4: 1$. This result is perhaps unusual, since a much wider range of $x_{0}$ is common in the Bacillus group, and indeed we need not expect the Koch \& Schaechter hypothesis, still less our restrictive assumptions, to apply directly to whole multicellular organisms.

Powell \& Errington (1963b) measured the volumes of Aerobacter aerogenes (Hinshelwood strain) growing in a defined medium at two temperatures. The growth rate was limited to $0.2 \mathrm{hr}^{-1}$ in a continuous culture. The ratios of extreme volumes in the two series of measurements were $4 \cdot 0: 1,3 \cdot 9: 1$. On the other hand, Dr J. R. Postgate (private communication) isolated a variant of the Hinshelwood strain which was apparently stable under similar conditions, when Powell \& Errington (1963 $b$ ) found the ratio of its extreme volumes to be $12 \cdot 5: 1$. It seems likely, however, that the restrictive conditions of continuous culture tend to make for uniformity of size. This can be true of so unpromising a species as Proteus vulgaris. Powell \& Errington (unpublished) grew the organism in continuous culture on a chemically defined medium ('H12P*'; Powell \& Errington, 1963a) at growth rates of 0.28 and $0.62 \mathrm{hr}^{-1}$. The corresponding ratios of extreme volumes were $3 \cdot 3: 1,2 \cdot 8: 1$. 
Errington, Powell \& Thompson (unpublished) measured the lengths at inception $\left(x_{i}\right)$ and termination $\left(x_{t}\right)$ of Escherichia coli, Pseudomonas aeruginosa and Serratia marcescens in four groups of experiments. In each of three groups, the ranges of $x_{i}$ and $x_{t}$ were less than $2: 1$, the greatest $x_{t}$ was less than four times the least $x_{i}$ and the least $x_{t}$ was greater than the greatest $x_{i}$. In the fourth group, the limits were exceeded only by the $x_{i}$ and $x_{t}$ associated with a single fission.

Apart from those of Collins \& Richmond, the above data were obtained from rather small samples (about 100 organisms). It is to be remembered that the expected range of a sample is less than that of the population from which it is drawn by an amount which is greater, the smaller the sample size.

Further evidence can be obtained from an examination of the range of generation times, $\tau$. Under the Koch \& Schaechter hypothesis and the simplifying assumptions,

$$
\begin{aligned}
0 & \leqslant \tau \leqslant \frac{1}{\nu} \log \left\{\frac{X /\left(\frac{1}{2}+\epsilon\right)}{X\left(\frac{1}{2}-\epsilon\right)}\right\} \\
& =-\nu^{-1} \log \left(\frac{1}{4}-\epsilon^{2}\right) \\
& =-2 \nu^{-1} \log \left\{\left(\frac{1}{4}-\epsilon^{2}\right)^{t}\right\} .
\end{aligned}
$$

Now, by (30), $\nu=-\mu_{1}^{\prime}(\log p) / \tau$, so

$$
0 \leqslant \tau \leqslant 2 \bar{\tau} \frac{-\log \left\{\left(\frac{1}{4}-\epsilon^{2}\right)^{\overline{1}}\right\}}{-\mu_{1}^{\prime}(\log p)} .
$$

But, since the distribution of $p$ is closely concentrated round the value $\frac{1}{2}$, the geometric mean of $p$ is greater than the geometric mean of its extremes, $\left(\frac{1}{4}-\epsilon^{2}\right)^{\frac{1}{1}}$. That is

$$
\begin{aligned}
\exp \left\{\mu_{1}^{\prime}(\log p)\right\} & >\left(\frac{1}{4}-\epsilon^{2}\right)^{\underline{1}} ; \\
\mu_{1}^{\prime}(\log p) & >\log \left\{\left(\frac{1}{4}-\epsilon^{2}\right)^{1}\right\} .
\end{aligned}
$$

Both members of the last inequality are negative, hence the last member of (38) is greater than $2 \bar{\tau}$ : the admissible range of $\tau$ is from 0 to a little more than $2 \bar{\tau}$. Information about the range of $\tau$ in real cultures is less cogent than information about the range of size, because (38) is a necessary but not sufficient condition for the truth of the simplifying assumption; however, there is a great deal more of it.

The inequality

$$
0 \leqslant \tau \leqslant 2 \bar{\tau}
$$

is evidently satisfied by the examples in Schaechter et al. (1962). Powell (1958) measured in all about 6000 generation times in 16 groups of experiments. Reference to the original records showed that, omitting non-viable organisms, only 7 of the individual $\tau$ exceeded twice the mean for the group to which they belonged; in the least favourable group, 2 of 367 generation times did not satisfy the inequality. Powell \& Errington's (1963a) data gave a similar ratio of 8 exceptions among 5000; at worst 4 in a group of 963 measurements.

Thus the required restrictions on the range of $x_{i}, x_{t}, x_{0}$ and $\tau$ are most strikingly met, to within a little, by many cultures of Gram-negative organisms.

\section{The critical size}

We introduced a critical size $X$ in the first place to simplify the mathematical relationships implied by the Koch \& Schaechter hypothesis. It is remarkable that real cultures so nearly satisfy the conditions which make the simplified formulae 
valid. We might perhaps identify $X$ with Koch \& Schaechter's critical size $\bar{c}$; it is not essential to the hypothesis that $\bar{c}$ should in any sense be a mean of the observed size of organisms at termination. It will be, as I think, at least temporarily profitable to consider the relation of $X$ to a more fundamental critical size which we may call $\Xi$ : this is the absolute minimum size at which an organism of a given species can divide into two viable daughters; a size determined by the minimum complement of enzymes, nuclear material and mechanical structural elements. The size $\Xi$ may be attained under the starvation conditions of a continuous culture run at a low dilution rate (D. Herbert \& D.W. Tempest, private communication; see also Herbert, 1958); under less arduous conditions we can expect the lower observed limit, $X$, to be larger than $\Xi$ and less clearly defined.

We can then, following Koch \& Schaechter, suppose that fission succeeds the attainment of the size $X$ after a variable interval determined by secondary causes. That the size $2 X$ is rarely exceeded means that organisms which fail to divide before four nuclei have developed are themselves rare, as the experiments of Schaechter et al. (1962) suggest.

\section{REFERENCES}

AdolPH, E. F. \& BAYNE-JonEs, S. (1932). Growth in size of micro-organisms measured from motion pictures. II. Bacillus megatherium. J. cell. comp. Physiol. 1, 409.

BAYNE-Jones, S. \& ADOLPH, E. F. (1932). Growth in size of micro-organisms measured from motion pictures. III. Bacterium coli. J. cell. comp. Physiol. 2, 329.

Collins, J. F. \& Richmond, M. H. (1962). Rate of growth of Bacillus cereus between divisions. J. gen. Microbiol. 28, 15.

ERDÉLY, A. (1954). Tables of Integral Transforms, vol. I. New York: McGraw-Hill Book Co. Inc.

Herbert, D. (1958). Some principles of continuous culture. In Recent Progress in Microbiology. Ed. Tunevall, G. Stockholm: Almqvist and Wiksell.

KENDALL, D. G. (1948). On the role of a variable generation time in the development of a stochastic birth process. Biometrika, 35, 316.

KосH, A. L. \& SchaEchter, M. (1962). A model for statistics of the cell division process. J. gen. Microbiol. 29, 435.

Powel, E. O. (1955). Some features of the generation times of individual bacteria. Biometrika, 42, 16.

Powell, E. O. (1956). Growth rate and generation time of bacteria, with special reference to continuous culture. J. gen. Microbiol. 15, 492.

Powell, E. O. (1958). An outline of the pattern of bacterial generation times. J. gen. Microbiol. 18, 382.

Powell, E. O. \& Errington, F. P. (1963a). Generation times of individual bacteria: some corroborative measurements. J. gen. Microbiol. 31, 315.

Poweil, E. O. \& Errington, F. P. $(1963 b)$. The size of bacteria, as measured with the Dyson image-splitting eyepiece. J.R. micr. Soc. 82, 39.

RAHN, O. (1932). A chemical explanation of the variability of the growth-rate. J. gen. Physiol. 15, 257.

Schaechter, M., Williamson, J. P., Hood, J. R. \& Koch, A. L. (1962). Growth, cell and nuclear divisions in some bacteria. J. gen. Microbiol. 29, 421. 